\section{Fiber ring laser sensor for strain-temperature discrimination based on four-wave mixing effect}

\author{
O. Frazão, ${ }^{a}$ R. Morais, ${ }^{a, b}$ J. M. Baptista, ${ }^{a, c}$ and \\ J. L. Santos ${ }^{a, d}$ \\ aINESC Porto, Rua do Campo Alegre, 687, 4169-007 \\ Porto, Portugal \\ E-mail: ofrazao@inescporto.pt \\ bSIEMENS, S.A., Rua Irmãos Siemens, 1 Ed. 1, Piso 0, \\ Siemens SA, IC - WON Alfragide 2720-093, \\ Amadora Portugal \\ 'Universidade da Madeira, Dep. de Matemática e \\ Engenharias, Campus da Penteada, 9000-390 Funchal, \\ Portugal \\ dUniversidade do Porto, Departamento de Física, Rua do \\ Campo Alegre, 687, 4169-007 Porto, Portugal
}

\begin{abstract}
We present a new configuration based on a fiber ring laser sensor with four-wave mixing effect generation for simultaneous measurement of strain and temperature. The fiber ring laser sensor, corresponding to the signal wavelength, is based on an erbium-doped fiber amplifier, a highly nonlinear bismuth-based optical fiber, and a Bragg grating filter. Four-wave mixing is generated when an external pump laser is combined with a ring laser sensor. Two new peaks appear and are named the converted and satellite wavelengths. With efficiency conversion, which corresponds to the optical power ratio between the converted signal and ring laser input signal, and a Bragg wavelength it is possible to discriminate strain and temperature. Maximum errors of $\pm 1.8^{\circ} \mathrm{C}$ and $\pm 12 \mu \varepsilon$ are reported over $100^{\circ} \mathrm{C}$ and $1000 \mu \varepsilon$ measurement ranges, respectively. (c) 2007 Society of PhotoOptical Instrumentation Engineers.

[DOI: 10.1117/1.2406065]
\end{abstract}

Subject terms: fiber optic sensors; fiber Bragg gratings; simultaneous measurements.

Paper 060602LR received Aug. 4, 2006; revised manuscript received Oct. 30, 2006; accepted for publication Nov. 7, 2006; published online Jan. 8, 2007.

\section{Introduction}

In the last several years, the use of fiber Bragg gratings as sensing elements and mostly for simultaneous measurement of strain and temperature has had a great evolution in several applications. However, a significant limitation of fiber Bragg gratings is their high intrinsic temperature crosssensitivity. The simplest way of measuring strain and temperature is to use physically separated sensing elements, where one of them is isolated from strain and the second one has an interaction with the two physical parameters. Considering this and in order to discriminate these two physical parameters, the sensing heads based in this technology use two serial Bragg gratings. Writing of fiber Bragg gratings in optical fibers with different wavelengths, ${ }^{1}$ diameters, ${ }^{2}$ doping, ${ }^{3}$ or others features ${ }^{4}$ are examples proposed by researchers in the last few years. These configurations may present some unfavourable aspects such as: the

0091-3286/2007/\$25.00 @ 2007 SPIE use of two pieces of equipment to interrogate the gratings ${ }^{1}$ and difficulty in writing fiber Bragg gratings in special fibers. ${ }^{2,3}$ In order to solve these problems, several researchers proposed the use of a single structure bearing two signatures, measuring other parameters besides wavelength such as the optical power ${ }^{5}$ and/or bandwidth. ${ }^{6}$ However, interrogating these sensing heads may carry other sort of difficulties, since the signatures may be too close and problems due to source fluctuations are likely to occur.

A research area within optical fiber sensing comprehends the nonlinear effects generated in optical fibers that prove to be sensitive to strain and temperature. These effects combined with a Bragg grating structure permit to discrimination of strain and temperature. In 1997, Davis and Kersey ${ }^{7}$ presented the use of the Brillouin effect and a Bragg grating to discriminate these two physical parameters. The resolution obtained was $22 \mu \varepsilon$ and $1.9^{\circ} \mathrm{C}$. Recently, a long-haul system for simultaneous measurement of strain and temperature using a Bragg grating combined with the Raman effect was presented. ${ }^{8}$ The resolution was $0.7^{\circ} \mathrm{C}$ and $8.64 \mu \varepsilon$ for a temperature range of $70^{\circ} \mathrm{C}$ and a strain range of $1800 \mu \varepsilon$.

This work proposes and demonstrates the use of a fourwave mixing (FWM) effect to interrogate a single Bragg grating structure in a ring laser to discriminate strain and temperature. The generation of the nonlinear effect is obtained due to the highly nonlinear bismuth-based optical fiber that is illuminated by the combination of the fiber ring laser sensor and external pump laser diode with distinct wavelengths. The discrimination of both physical parameters is assured when we use the Bragg wavelength combined with the linear region of maximum FWM efficiency. Comparing both physical parameter responses, a high differential sensitivity and a relatively small condition number were observed.

\section{Experimental Details}

\subsection{Optical Fiber Characteristics}

The nonlinear fiber used in this experiment is a bismuth oxide $\left(\mathrm{Bi}_{2} \mathrm{O}_{3}\right)$ based optical fiber (Bi-NLF) kindly supplied by Asahi Glass Co. Ltd. The nonlinear coefficient of this fiber is $\gamma=1360 \mathrm{~W}^{-1} \mathrm{~km}^{-1}$, its length $L=2.6 \mathrm{~m}$, the attenuation $\alpha=2.2 \mathrm{~dB} / \mathrm{m}$, and the chromatic dispersion $D_{c}=-270 \mathrm{ps} \mathrm{nm}^{-1} \mathrm{~km}^{-1}$.

A traditional experimental setup was used for measuring the FWM efficiency of the Bi-NLF that corresponds to the ratio between the optical power of the conversion signal and the optical power of the pump signal. At fiber input, pump power was $20.23 \mathrm{dBm}$ and signal power was $2.76 \mathrm{dBm}$. Polarizations were aligned to maximize FWM interaction and the signal wavelength was varied in $0.1 \mathrm{~nm}$ steps. The resulting power of the FWM converted signal is represented in Fig. 1. The FWHM bandwidth is approximately $2.5 \mathrm{~nm}$. This value is acceptable for a sensing head, corresponding to a range of $250^{\circ} \mathrm{C}$ and $2500 \mu \varepsilon$ for temperature and strain measurement. Nevertheless, Lee et al. ${ }^{9}$ demonstrated the FWM effect using $40 \mathrm{~cm}$ of the same fiber and the reported bandwidth was approximately $6 \mathrm{~nm}$. Due to high nonlinear coefficient and low dispersion slope, this FWM bandwidth is approximately constant within the entire $\mathrm{C}$ band $(1530-1565 \mathrm{~nm})$. 


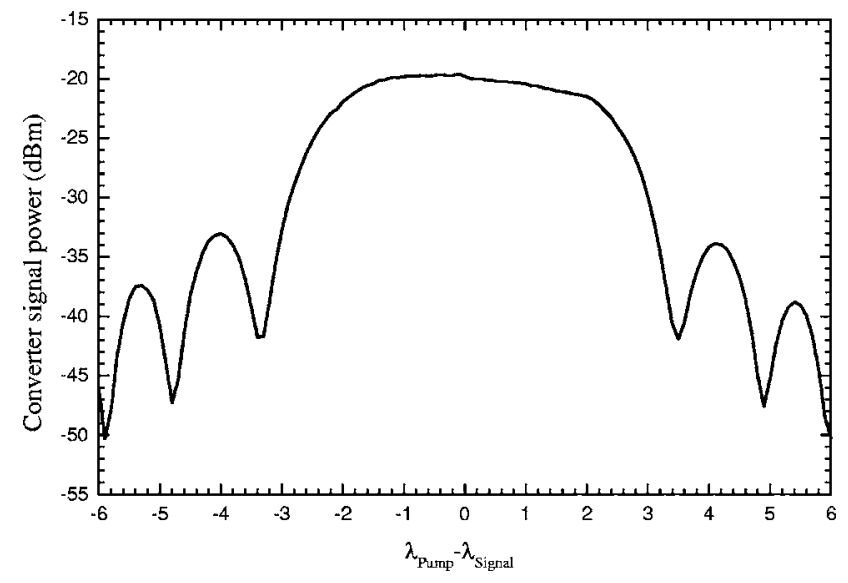

Fig. 1 Four-wave mixing efficiency using Bi-NLF.

\subsection{Experimental Results}

Figure 2 shows the proposed fiber ring resonator that incorporates a $3 \mathrm{~dB}$ optical coupler, the Bi-NLF, and an optical amplifier with up to $30 \mathrm{dBm}$ of output power. The Bragg grating sensor is connected to one of the coupler output ports. The sensor was fabricated using the following fiber: Corning SMF28 standard single-mode optical fiber, germanosilicate core with $3 \mathrm{~mol} \%$ of $\mathrm{GeO}_{2}$, coldhydrogenated at $100 \mathrm{~atm}$. The Bragg wavelength of the FBG sensor is $1548.7 \mathrm{~nm}$ with $\sim 100 \%$ reflectivity. In the other coupler output port a pump laser diode with $10 \mathrm{~mW}$ of optical power at $1548.2 \mathrm{~nm}$ was connected through an optical circulator. All measurements were recorded using an optical spectrum analyser with $0.05 \mathrm{~nm}$ of resolution. The sensing head was bonded to a translation stage (TS) with a displacement resolution of $1 \mu \mathrm{m}$ and placed in a tubular furnace, with a resolution of $0.1^{\circ} \mathrm{C}$.

The response to temperature and strain of the grating sensor was determined separately. Figure 3 shows the experimental results obtained when temperature was fixed to $20^{\circ} \mathrm{C}$ and strain varied up to $1000 \mu \varepsilon$. The strain coefficients of the wavelength peak and efficiency conversion are shown in Table 1. Figure 4 presents the temperature results when the strain was fixed and the temperature varied between $20^{\circ} \mathrm{C}$ to $100^{\circ} \mathrm{C}$. Table 2 shows the temperature coefficients.

The signal wavelength $\left(\lambda_{\text {signal }}\right)$ and the efficiency conversion $(\eta)$ were used and applied in the matrix method. ${ }^{4}$

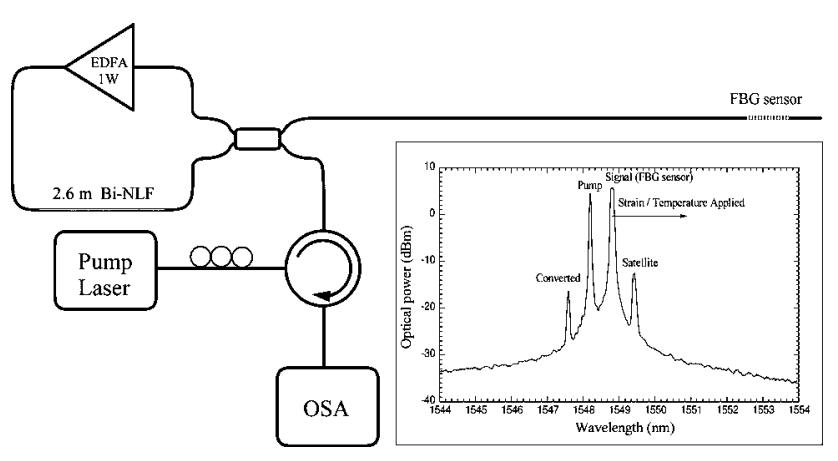

Fig. 2 Fiber ring laser sensor.

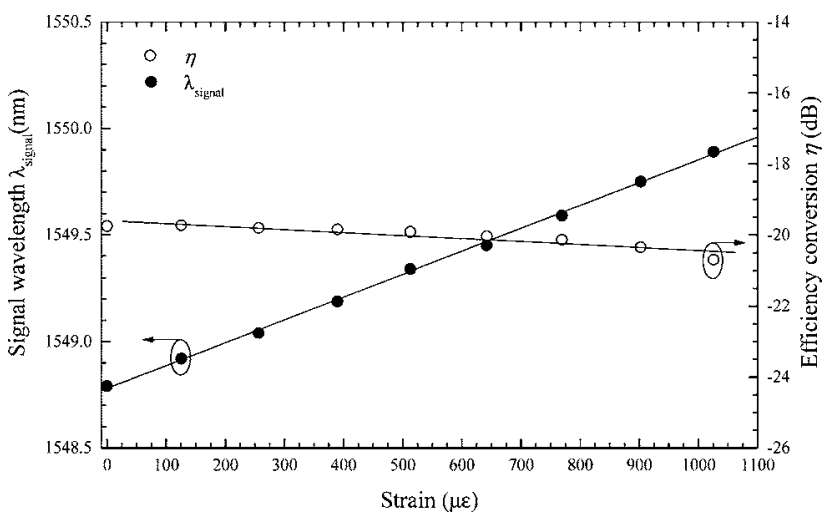

Fig. 3 Response of the fiber laser sensor when temperature is fixed and strain is varied.

The matrix method can be used to determine the $N$ parameters to which the sensor head is sensitive. However, this is only possible if $N$ different characteristics of the sensing head structure can be independently determined, and such characteristics change differently under the action of the $N$ measurands. In a particular case where the sensor head has linear sensitivities for all parameters, it is possible to write $N$ independent equations allowing explicit solutions for the actual value of each measurand, even in the situation where all of them are changing. This concept is better illustrated in the case where only two measurands are considered, for instance, the strain-temperature discrimination, which will be addressed hereafter.

Strain and temperature can be obtained simultaneously using the following matrix equation:

$\left[\begin{array}{c}\Delta T \\ \Delta \varepsilon\end{array}\right]=\frac{1}{D}\left[\begin{array}{cc}K_{\varepsilon(\eta)} & -K_{\varepsilon(\text { signal })} \\ -K_{T(\eta)} & K_{T(\text { signal })}\end{array}\right]\left[\begin{array}{c}\Delta \lambda_{\text {signal }} \\ \Delta \eta\end{array}\right]$

where $D=K_{T(\text { signal })} K_{\varepsilon(\eta)}-K_{\varepsilon(\text { signal })} K_{T(\eta)}$. The strain and temperature coefficients $K_{\varepsilon}$ and $K_{T}$, are obtained by fitting the experimental data using the linear regression presented in Figs. 3 and 4.

In such conditions, Eq. (1) can be used in order to have an explicit matrix equation that allows simultaneous measurement of $\Delta T$ and $\Delta \varepsilon$ for this sensing head:

$\left[\begin{array}{c}\Delta T \\ \Delta \varepsilon\end{array}\right]=-\frac{1}{2.04}\left[\begin{array}{cc}-0.84 & -1.08 \\ 5.89 & 10.01\end{array}\right]\left[\begin{array}{c}\Delta \lambda_{\text {signal }} \\ \Delta \eta\end{array}\right]$.

In this case $D$ is small, and it is thus important to study the stability of the matrix. The stability can be calculated through the matrix condition number. The matrix condition number is defined as $K(\Omega)=\|\Omega\|\left\|\Omega^{-1}\right\|$, where \|\| is the matrix norm induced by a vector norm and $\Omega$ corresponds to

Table 1 Strain coefficients.

Designation

$\left(K_{\varepsilon}\right)$

Signal (FBG sensor)

$1.08 \times 10^{-3} \mathrm{~nm} / \mu \varepsilon$

Efficiency conversion $(\eta)$

$-0.84 \times 10^{-3} \mathrm{~dB} / \mu \varepsilon$ 


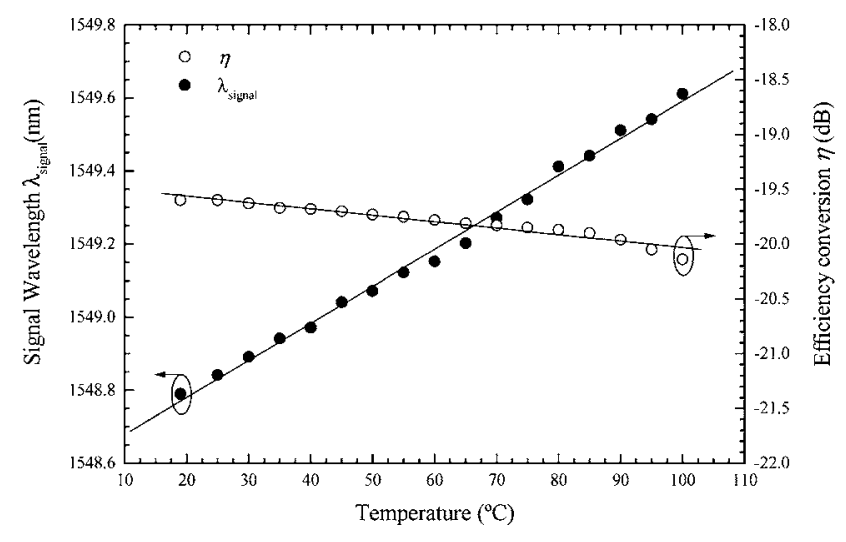

Fig. 4 Response of the fiber laser sensor when strain is fixed and temperature is varied.

the matrix with the sensitivity coefficients. If the condition number is very high $(\gg 1)$, then a small error in $\Delta \lambda$ will introduce a large error in physical parameter $\Delta \mathrm{T}$ and $\Delta \varepsilon$. In Eq. (2) the matrix condition number is 66.79 . This value is satisfactory, resulting in an acceptable conditioned evaluation matrix. Sivanesan et al. ${ }^{10}$ present the condition numbers for different dual-fiber grating sensor systems according to the reported matrix-element values. The condition number obtained in this work is small compared with the conventional sensing head based on two Bragg grating structures. See Table 3.

The performance of the matrix was evaluated when the sensing head undertook strain variations in a range of $1000 \mu \varepsilon$ at a fixed temperature $\left(T=50^{\circ} \mathrm{C}\right)$ and the other way around, i.e., temperature variations in a range of $100^{\circ} \mathrm{C}$ for a specific applied strain $(\varepsilon=500 \mu \varepsilon)$. The results have maximum errors of $\pm 1.8^{\circ} \mathrm{C}$ and $\pm 12 \mu \varepsilon$, for temperature and strain, respectively. The maximum errors include the errors that come from the use of the linear regression fit, and the errors from the use of the matrix method.

\section{Conclusions}

We have demonstrated a new configuration based on a fiber ring laser sensor with the generation of a four-wave mixing effect for simultaneous measurement of strain and temperature. An advantage of this configuration is the use of only one Bragg grating structure when compared with a traditional sensing head where two serial Bragg grating structures are used. Another advantage is the high differential sensitivity resulting in a relatively small condition number. On the other hand and due to the generated high power, this

Table 2 Temperature coefficients.

\begin{tabular}{lc}
\hline \hline Designation & $\left(K_{T}\right)$ \\
\hline Signal (FBG sensor) & $10.01 \times 10^{-3} \mathrm{~nm} /{ }^{\circ} \mathrm{C}$ \\
Efficiency conversion $(\eta)$ & $-5.89 \times 10^{-3} \mathrm{~dB} /{ }^{\circ} \mathrm{C}$ \\
\hline \hline
\end{tabular}

Table 3 Condition numbers of different dual grating sensors. ${ }^{10}$

\begin{tabular}{lc}
\hline \hline Dual grating method & $K(\Omega)$ \\
\hline Two superimposed gratings & 123.6 \\
Two different dopings & 147.9 \\
$\begin{array}{l}\text { First- and second-order resonances } \\
(1561 \mathrm{~nm}, 789 \mathrm{~nm})\end{array}$ & 203.29 \\
$\begin{array}{l}\text { First- and second-order resonances } \\
(1300 \mathrm{~nm}, 660 \mathrm{~nm})\end{array}$ & 160.78 \\
\hline \hline
\end{tabular}

sensing configuration enables the sensor head to be remotely located without affecting the FWM response. As a possible future improvement, the interrogation system may also be able to multiplex several sensor heads by using a $1 \times N$ optical switch, placed between the Bragg grating sensor and the optical coupler.

\section{Acknowledgments}

The authors would like to thank Dr. Naoki Sugimoto and Asahi Glass Co. Ltd. for supplying the bismuth oxide based fiber.

This work was supported in part by "Fundação para a Ciência e Tecnologia" under the programme "Programa Operacional Ciência Tecnologia e Inovação"-POCTI/ FEDER with grant REEQ/1272/EEI/2005 Fiber Optic Supported Broadband Communication Networks.

\section{References}

1. M. G. Xu, J-L. Archambault, L. Reekie, and J. P. Dakin, "Discrimination between strain and temperature effects using dual-wavelength fiber grating sensors," Electron. Lett. 30(13), 1085-1087 (1994).

2. S. W. James, M. L. Dockney, and R. P. Tatam, "Simultaneous independent temperature and strain measurement using in-fiber Bragg grating sensors," Electron. Lett. 32(12), 1133-1134 (1996).

3. P. M. Cavaleiro, F. M. Aráujo, L. A. Ferreira, J. L. Santos, and F. Farahi, "Simultaneous measurement of strain and temperature using Bragg gratings written in germanosilicate and boron-Codoped germanosilicate fibers," IEEE Photonics Technol. Lett. 11(12), 1635-1637 (1999).

4. O. Frazão, L. A. Ferreira, F. M. Araujo, and J. L. Santos, "Applications of fiber optic grating technology to multi-Parameter measurement," Fiber Integr. Opt. 24(3-4), 227-244 (2005).

5. O. Frazão, R. Romero, G. Rego, P. V. S. Marques, H. M. Salgado, and J. L. Santos, "Sampled fiber Bragg grating sensors for simultaneous strain and temperature measurement," Electron. Lett. 38(14), 693-695 (2002).

6. O. Frazão, R. Romero, F. M. Araújo, L. A. Ferreira, and J. L. Santos, "Strain-temperature discrimination using a step spectrum profile fiber Bragg grating arrangement," Sens. Actuators, A 120, 490-499 (2005).

7. M. A. Davis and A. D. Kersey, "Simultaneous measurement of temperature and strain using fiber Bragg gratings and Brillouin scattering," IEE Proc.: Optoelectron. 144(3), 151-155 (1997).

8. J. H. Lee, Y. M. Chang, Y-G. Han, H. Chung, S. H. Kim, and S. B. Lee, "Raman amplifier-based long-distance remote, strain and temperature sensing system using an erbium-doped fiber and a fiber Bragg grating," Opt. Express 12(12), 2774 (2004).

9. J. H. Lee, T. Nagashima, T. Hasegawa, S. Ohara, N. Sugimoto, and K. Kikuchi, "Four-wave-mixing-based wavelength conversion of 40$\mathrm{Gb} / \mathrm{s}$ nonreturn-to-zero signal using 40-cm bismuth oxide nonlinear optical fiber," IEEE Photonics Technol. Lett. 17(7), 1474-1476 (2005).

10. P. Sivanesan, J. S. Sirkis, Y. Murata, and S. G. Buckley, "Optimal wavelength pair selection and accuracy analysis of dual fiber grating sensors for simultaneously measuring strain and temperature," Opt. Eng. 41(10), 2456-2463 (2002). 